\title{
Characterization of a 62-Kilodalton Acidic Phospholipid-Binding Protein Isolated from the Edible Mushroom Pleurotus ostreatus
}

\author{
Hideko Tanaka and Tetsuyuki Kobayashi* \\ Department of Biological Sciences, Graduate School of Humanities and Sciences, Ochanomizu University, 2-1-1 Ohtsuka, Bunkyo-ku, \\ Tokyo 112-8610, Japan
}

(Received October 31, 2010; Accepted November 2, 2010; Published online November 10, 2010)

Many lipid-binding proteins such as pleurotolysin and ostreolysin have been isolated from the edible mushroom Pleurotus ostreatus. In this study, we detected a novel lipid-binding protein with a molecular weight of $62 \mathrm{kDa}$ by measuring via centrifugation the association of aqueous extracts of the mushroom with lipid vesicles composed of various phospholipids. The 62-kDa protein (p62) was purified by sedimentation of the mixture of protein extracts and acidic phospholipid-containing lipid vesicles. The purified $\mathbf{p 6 2}$ bound to phosphatidylglycerol (PG)/phosphatidylcholine/cholesterol (5 : 45 : 50) vesicles but not to vesicles composed of other phospholipids including phosphatidylserine (PS), phosphatidylinositol, phosphatidic acid, lysoPS, and lysophosphatidylinositol. The $\mathbf{p} 62$ protein specifically associated with the PG-containing vesicles but not with other polyglycerophospholipid vesicles consisting of cardiolipin, bis(monoacylglycero)phosphate, monolysocardiolipin, or dilysocardiolipin, suggesting that $\mathbf{p 6 2}$ recognized a precise molecular structure of PG. Intrinsic tryptophan fluorescence of $\mathrm{p62}$ was changed by incubation of $\mathbf{p 6 2}$ with PG-containing vesicles. Staining of giant unilamellar vesicles with fluorescence-labeled p62 showed that $\mathrm{p62}$ bound to PG-containing vesicles but not PS-containing vesicles. These observations signify the potential usefulness of $\mathbf{p 6 2}$ as a tool for studying the functions of PG molecules in biological membranes.

Key words — Pleurotus ostreatus, lipid-binding protein, phosphatidylglycerol, acidic phospholipid, mushroom

\footnotetext{
${ }^{*}$ To whom correspondence should be addressed: Department of Biological Sciences, Graduate School of Humanities and Sciences, Ochanomizu University, 2-1-1 Ohtsuka, Bunkyo-ku, Tokyo 112-8610, Japan. Tel. \& Fax: +81-3-5978-2604; Email: kobayashi.tetsuyuki@ocha.ac.jp
}

\section{INTRODUCTION}

Little is known about where particular phospholipids are localized in membranes and how they participate in cellular functions, mainly because of the lack of appropriate methodologies for manipulating and tracing the functions of membrane phospholipids. Various probes for studying the molecular motion of membrane phospholipids have been developed, such as lysenin specific to sphingomyelin (SM), ${ }^{1-4)}$ a small cyclic peptide specific to phosphatidylethanolamine, ${ }^{5,6)}$ and monoclonal antibodies against phosphatidylcholine (PC), ${ }^{7)}$ phosphatidylserine (PS), ${ }^{8,9)}$ and phosphatidylinositol 4,5-bisphosphate. ${ }^{10,11)}$

Several cytolytic proteins have also been isolated from the basidiocarps of both toxic and edible mushrooms, and their lipid-binding specificities as well as pore-forming properties have been studied. ${ }^{12-16)}$ A 2-component hemolysin, pleurotolysin A (17 kDa) and pleurotolysin B $(59 \mathrm{kDa})$ from Pleurotus ostreatus (P. ostreatus), has been purified and characterized. ${ }^{13,17)}$ Pleurotolysin A specifically bound to SM/cholesterol vesicles and formed a transmembrane pore in concert with pleurotolysin B. Ostreolysin was also isolated as a 16$\mathrm{kDa}$ hemolytic protein from the same mushroom, which was suggested to specifically recognize a cholesterol-rich lipid domain. ${ }^{18-20)}$

In the present study, we detected a $62-\mathrm{kDa}$ protein (p62) from P. ostreatus and showed that p62 binds to lipid vesicles containing acidic phospholipids, especially phosphatidylglycerol (PG).

\section{MATERIALS AND METHODS}

Materials — Egg yolk PC, 1-palmitoyl-2-oleoyl 
PC (POPC), egg yolk phosphatidylethanolamine, bovine brain SM, bovine brain PS, bovine liver phosphatidylinositol, egg yolk phosphatidic acid, egg yolk PG, and bovine heart cardiolipin were purchased from Sigma (St. Louis, MO, U.S.A.). Bis(monoacylglycero)phosphate, monolysocardiolipin, and dilysocardiolipin were kindly provided by Dr. T. Kobayashi of RIKEN (Wako, Saitama, Japan). Cholesterol, lysoPS, and lysophosphatidylinositol were purchased from Avanti Polar Lipids (Alabaster, AL, U.S.A.). 3-[(3-Cholamidopropyl) dimethylammonio]propanesulfonate (CHAPS) was obtained from Dojindo Laboratories (Tokyo, Japan). A fluorescein isothiocyanate (FITC) labeling kit was purchased from Thermo Fisher Scientific K.K. (Yokohama, Japan). All other chemicals used in this study were of analytical grade.

Purification of p62 - All purification steps were carried out at $0-4^{\circ} \mathrm{C}$, and p 62 fractions were treated with protease inhibitors at each step. Basidiocarps of P. ostreatus (100 g, wet weight) were homogenized in $100 \mathrm{ml}$ of $50 \mathrm{mM}$ Tris-HCl buffer ( $\mathrm{pH} 8.5$ ). The homogenate was centrifuged at $15000 \times g$ for $30 \mathrm{~min}$, and ammonium sulfate was added to the supernatant to $35 \%$ saturation. After $30 \mathrm{~min}$, the mixture was centrifuged at $15000 \times g$ for $30 \mathrm{~min}$, and ammonium sulfate was added to the supernatant to $70 \%$ saturation. The resultant precipitates were dialyzed against $50 \mathrm{mM}$ Tris- $\mathrm{HCl}$ buffer ( $\mathrm{pH} 8.5$ ).

Lipid vesicles composed of PS and cholesterol ( $1: 1$, molar ratio; $0.1 \mathrm{mmol}$ of lipids) were incubated with the ammonium sulfate fraction proteins (100 $\mathrm{mg}$ of proteins precipitating between $35-70 \%$ saturation) in $20 \mathrm{ml}$ of $50 \mathrm{mM}$ Tris- $\mathrm{HCl}$ buffer $(\mathrm{pH}$ 8.5) containing $2 \mathrm{mM}$ EDTA at $4^{\circ} \mathrm{C}$ for $60 \mathrm{~min}$ on ice. The liposome fractions were collected by centrifugation at $16000 \times g$ for $10 \mathrm{~min}$ at $4^{\circ} \mathrm{C}$ and washed twice with $50 \mathrm{mM}$ Tris-HCl buffer $(\mathrm{pH} 8.5)$ containing $0.1 \%$ (w/v) CHAPS. The washed liposomes were solubilized in $0.5 \%$ (w/v) CHAPS at $37^{\circ} \mathrm{C}$ for $10 \mathrm{~min}$ and were centrifuged at $16000 \times$ $g$ for $30 \mathrm{~min}$. The supernatants were used as a source of the purified p62 protein in subsequent lipid-binding assays using sodium dodecyl sulfatepolyacrylamide gel electrophoresis (SDS-PAGE). The protein concentrations were determined spectrophotometrically by using the BCA Protein Assay Reagent (Thermo Fisher Scientific K.K.).

Binding Assay of P. ostreatus Proteins to Vesicles — The multilamellar vesicles consisting of $50 \mathrm{~mol} \% \mathrm{PC}, \mathrm{SM}$, PS, or phosphatidylinositol and
$50 \mathrm{~mol} \%$ cholesterol were prepared for the binding studies. Lipids that were in chloroform were placed in Pyrex glass tubes, and the organic solvent was removed by using a rotary evaporator. The lipid film was thoroughly dried to remove residual organic solvent by placing the samples on a vacuum pump overnight. The dried lipid film was dispersed in $100 \mu \mathrm{l}$ of Tris- $\mathrm{HCl}(\mathrm{pH} 8.5)$ at room temperature and agitated vigorously by using a vortex mixer. The test tubes containing the lipid suspension were placed in a bath sonicator and gently sonicated for $15 \mathrm{~min}$. The suspension was transferred to a microcentrifuge tube and centrifuged for $10 \mathrm{~min}$ at 16000 $\times g$. The supernatant was removed, and the pellet, resuspended in Tris- $\mathrm{HCl}$ ( $\mathrm{pH} 8.5$ ), was used for the binding studies. Each of the lipid vesicles was incubated with ammonium sulfate fraction proteins from $P$. ostreatus at $37^{\circ} \mathrm{C}$ for $30 \mathrm{~min}$. The vesicles were washed twice with Tris- $\mathrm{HCl}(\mathrm{pH} 8.5)$, and subjected to SDS-PAGE. Protein bands were stained with Coomassie brilliant blue R-250.

To assess the binding activity of purified p62 to acidic phospholipids in membranes, lipid vesicles composed of $5 \mathrm{~mol} \% \mathrm{PG}$, PS, phosphatidylinositol, lysoPS, lysophosphatidylinositol, or phosphatidic acid, $45 \mathrm{~mol} \%$ PC from egg yolk, and $50 \mathrm{~mol} \%$ cholesterol were prepared. Then, the binding assay was performed as described above.

The binding activities of p62 to the polyglycerophospholipids, namely bis(monoacylglycero)phosphate, monolysocardiolipin, and dilysocardiolipin in membranes were examined by incubating p62 with lipid vesicles composed of $10 \mathrm{~mol} \%$ polyglycerophospholipids, $40 \mathrm{~mol} \%$ egg yolk PC, and $50 \mathrm{~mol} \%$ cholesterol, which were prepared as described above.

Fluorescence Measurements — Steady-state intrinsic fluorescence of p62, either alone or in combination with lipids, was measured at $37^{\circ} \mathrm{C}$ in a Fluoromax spectrofluorimeter (HORIBA, Kyoto, Japan) equipped with a thermostatically controlled cell holder and a magnetic stirrer. Excitation and emission slits were set at $5 \mathrm{~nm}$ as described. ${ }^{2,3)}$ Samples were excited at $280 \mathrm{~nm}$. Fluorescence emission spectra of $5 \mu \mathrm{g} / \mathrm{ml}$ of p62 were recorded over the range of $300-400 \mathrm{~nm}$. Intrinsic tryptophan fluorescence signals were corrected for the dilution factor, and the background was subtracted by using appropriate blanks. Small unilamellar vesicles composed of $10 \mathrm{~mol} \% \mathrm{PG}$, PS, or cardiolipin, $40 \mathrm{~mol} \%$ $\mathrm{PC}$, and $50 \mathrm{~mol} \%$ cholesterol were prepared by $10-$ min pulsed sonication with a Sonifier (Branson Ul- 
trasinic Corp., Danbury, CT, U.S.A.), using output scale 4 and $50 \%$ duty cycle at room temperature. All the fluorescence measurements were taken in $50 \mathrm{mM}$ Tris-HCl buffer ( $\mathrm{pH} 8.5)$.

Preparation of Giant Unilamellar Vesicles and Observation of p62 Binding under Fluorescent Microscopy — The ratio between the components comprising the vesicles (PG/POPC/cholesterol or $\mathrm{PS} / \mathrm{POPC} /$ cholesterol) was fixed at 10:40:50. Giant unilamellar vesicles were prepared by the gentle hydration method as previously described. ${ }^{21}$ ) The vesicle suspension was incubated at room temperature with FITCconjugated p62, which was prepared as reported, ${ }^{22)}$ and then transferred to a glass plate. The samples were observed with an inverted fluorescence microscope.

\section{RESULTS}

\section{Detection of the Phospholipid-binding Proteins from $P$. ostreatus}

To detect the phospholipid-binding proteins, aqueous extracts of $P$. ostreatus (acquired from 35$70 \%$ ammonium sulfate fractionation) were incubated with lipid vesicles composed of SM, PC, PS, or phosphatidylinositol and cholesterol at a molar ratio of $1: 1$. After incubation at $37^{\circ} \mathrm{C}$ for $30 \mathrm{~min}$, the solutions were centrifuged. The resultant pellets containing lipid vesicle-associated proteins were subjected to SDS-PAGE (Fig. 1). A protein band with a molecular weight of $17 \mathrm{kDa}$, which corresponds to pleurotolysin, ${ }^{13,17)}$ was observed by sedimentation of the aqueous extracts with SM-containing vesicles. It was reported that pleurotolysin A specifically bound to SMcholesterol vesicles. ${ }^{13)}$ Interestingly, the pellets of acidic-phospholipid vesicles, namely PS and phosphatidylinositol, showed a single protein band with an apparent molecular weight of $62 \mathrm{kDa}$. In contrast, no protein band was observed with $\mathrm{PC}$ vesicles under the same conditions.

\section{Purification of p62 from $P$. ostreatus}

The protein $\mathrm{p} 62$ was purified from basidiocarps of $P$. ostreatus by ammonium sulfate precipitation, followed by sedimentation with lipid vesicles composed of PS and cholesterol at a 1:1 molar ratio. Efficient enrichment of p62 was observed by sedimentation of the P. ostreatus aqueous extract with the PS-cholesterol vesicle (Fig. 2, lane 2). The pellet

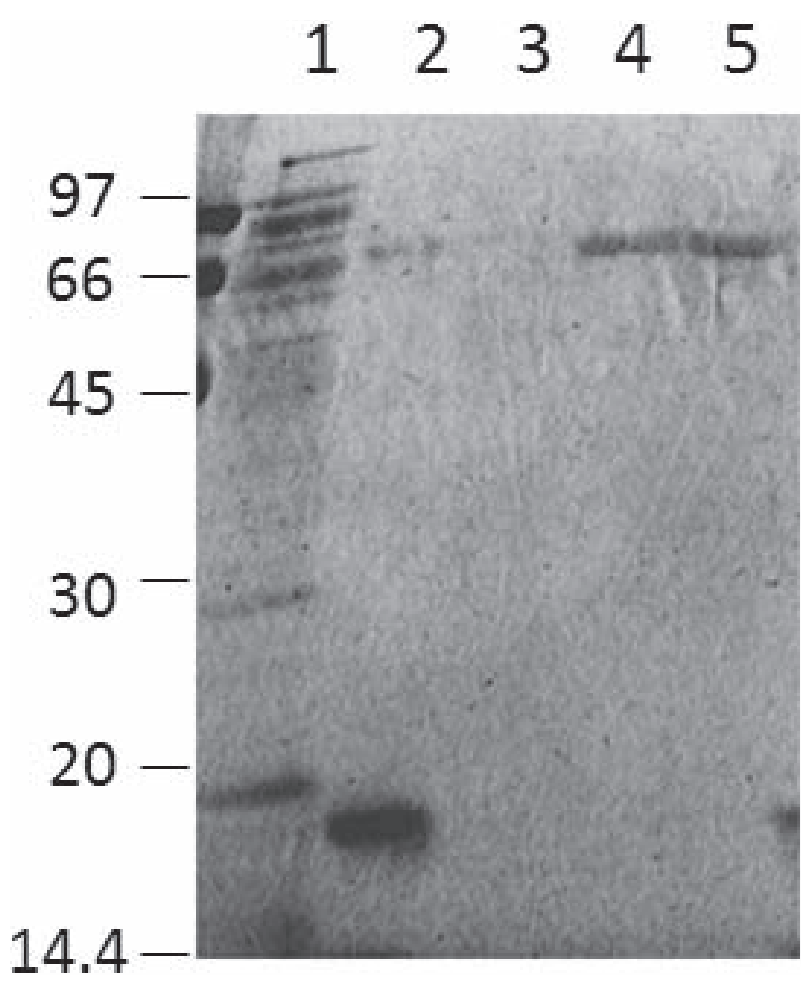

Fig. 1. SDS-PAGE of Lipid Vesicle-associated Proteins from P. ostreatus

P. ostreatus aqueous extracts separated by $35-70 \%$ ammonium sulfate fractionation were incubated with lipid vesicles composed of various phospholipids and cholesterol at $1: 1$ molar ratio at $37^{\circ} \mathrm{C}$ for $30 \mathrm{~min}$; SM/cholesterol (lane 2), PC/cholesterol (lane 3), PS/cholesterol (lane 4), or phosphatidylinositol/cholesterol (lane 5). The samples were centrifuged and the resulting pellets were analyzed by SDS-PAGE. Protein bands were stained with Coomassie brilliant blue R-250. The ammonium sulfate fraction from P. ostreatus is shown in lane 1 .

was washed with $0.1 \%$ CHAPS to remove nonspecific binding proteins, and purified p62 was further solubilized in $0.5 \%$ CHAPS (Fig. 2, lane 3). The supernatant was loaded onto a POROS ${ }^{\circledR}$ HQ anion exchange column to remove CHAPS.

Tryptic peptides of the p62 band were analyzed by matrix-assisted laser desorption/ionization timeof-flight mass spectrometry by using peptide mass matching. Mass spectra database search indicated that there were no high scoring homologous proteins. This nearly purified p62 was used for the subsequent experiments.

\section{Binding Specificity of p62 to Acidic Phospho- lipids}

The interaction of purified p62 with various acidic phospholipids was examined by measuring its association with lipid vesicles via centrifugation of the p62 extract mixed with vesicles containing $5 \mathrm{~mol} \%$ acidic phospholipids, $45 \mathrm{~mol} \% \mathrm{PC}$, and 


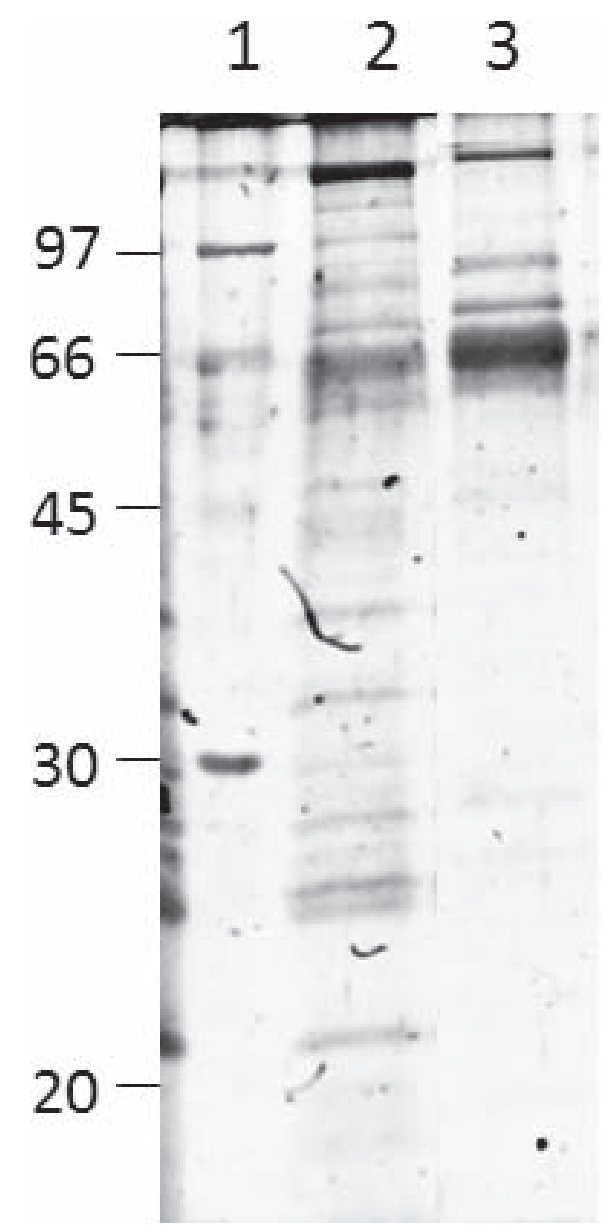

Fig. 2. Purification of p62 by Sedimentation with PS/cholesterol Vesicles

Lipid vesicles composed of PS and cholesterol (50:50, molar ratio) were incubated with ammonium sulfate fraction proteins (35$70 \%$ ) at $4^{\circ} \mathrm{C}$ for $60 \mathrm{~min}$. The samples were centrifuged to precipitate lipid vesicles. The pellets were washed twice with a buffer containing $0.1 \%$ CHAPS and further treated with $0.5 \%$ CHAPS to solubilize p62. The samples were electrophoresed on $12 \%$ SDS-PAGE, and protein bands were stained with Oriole fluorescent gel stain (Bio-Rad). Lane 1 , molecular weight marker; lane 2, pellet of lipid vesicles after incubation with $P$. ostreatus aqueous extract; lane 3, supernatant after $0.5 \%$ CHAPS elution of vesicle-bound proteins from vesicles.

$50 \mathrm{~mol} \%$ cholesterol. The amount of bound p62 in the lipid vesicle-containing pellet was determined by SDS-PAGE.

The p62 protein was efficiently recovered by sedimentation of the $P$. ostreatus aqueous extract with PG-containing vesicles, but not with other acidic phospholipids, namely PS, phosphatidylinositol, and phosphatidic acid (Fig. 3A). No association of p62 with monoacyl analogs of phospholipids such as lysoPS and lysophosphatidylinositol was observed. As shown in Fig. 3B, the binding of p62 with lipid vesicles that were composed of a variety of polyglycerophospholipids was also compared. The p62 protein specifically bound to the PG-containing vesicles but did not bind to other lipid vesicles containing cardiolipin, bis(monoacylglycero)phosphate, monolysocardiolipin, or dilysocardiolipin.

Intrinsic tryptophan fluorescence is widely used to estimate the local environment of tryptophan residues in proteins. We compared the fluorescence emission spectra of tryptophan in the absence or presence of one of the following liposomes: PG/PC/cholesterol, PS/PC/cholesterol, cardiolipin/PC/cholesterol, and PC/cholesterol (Fig. 4). The largest increase in tryptophan fluorescence and a slight blue shift from $356 \mathrm{~nm}$ to $354 \mathrm{~nm}$ in the maximum emission wavelength was observed after incubation with PG/PC/cholesterol vesicles. Moderate changes in fluorescence were observed in the presence of $\mathrm{PS} / \mathrm{PC} /$ cholesterol or cardiolipin/PC/cholesterol vesicles. These results suggest the migration of the residual tryptophans of p62 to a less polar environment after incubation with PG-containing vesicles.

The binding of fluorescence-labeled p62 to PG in giant unilamellar vesicles was also examined. After incubation of the PG/PC/cholesterol $(10: 40: 50)$ giant vesicles with FITC-labeled p62, vesicles tended to aggregate larger than the $\mathrm{PS} / \mathrm{PC} /$ cholesterol vesicles as shown in Fig. 5. In addition, PG/PC/cholesterol giant vesicles were labeled fluorescently by incubation with FITC-p62, whereas PS/PC/cholesterol vesicles were not. These observations suggest the potential usefulness of p62 as a tool for studying the functions of PG molecules in biological membranes.

\section{DISCUSSION}

In the present study, we detected a $17 \mathrm{kDa}$ protein, which corresponds to pleurotolysin A, by sedimentation with SM/cholesterol vesicles. Pleurotolysin is a SM-specific cytolysin that consists of $\mathrm{A}(17 \mathrm{kDa})$ and $\mathrm{B}(59 \mathrm{kDa})$ components from $P$. ostreatus. ${ }^{13,17)}$ It is known that pleurotolysin A alone specifically binds to SM/cholesterol vesicles, which is consistent with our data, and forms transmembrane pore complex in concert with pleurotolysin B. ${ }^{13)}$

The p62 protein was efficiently enriched as a lipid vesicle-associated protein by incubation of the P. ostreatus extract with PS/cholesterol $(50: 50)$ or phosphatidylinositol/cholesterol $(50: 50)$ vesi- 

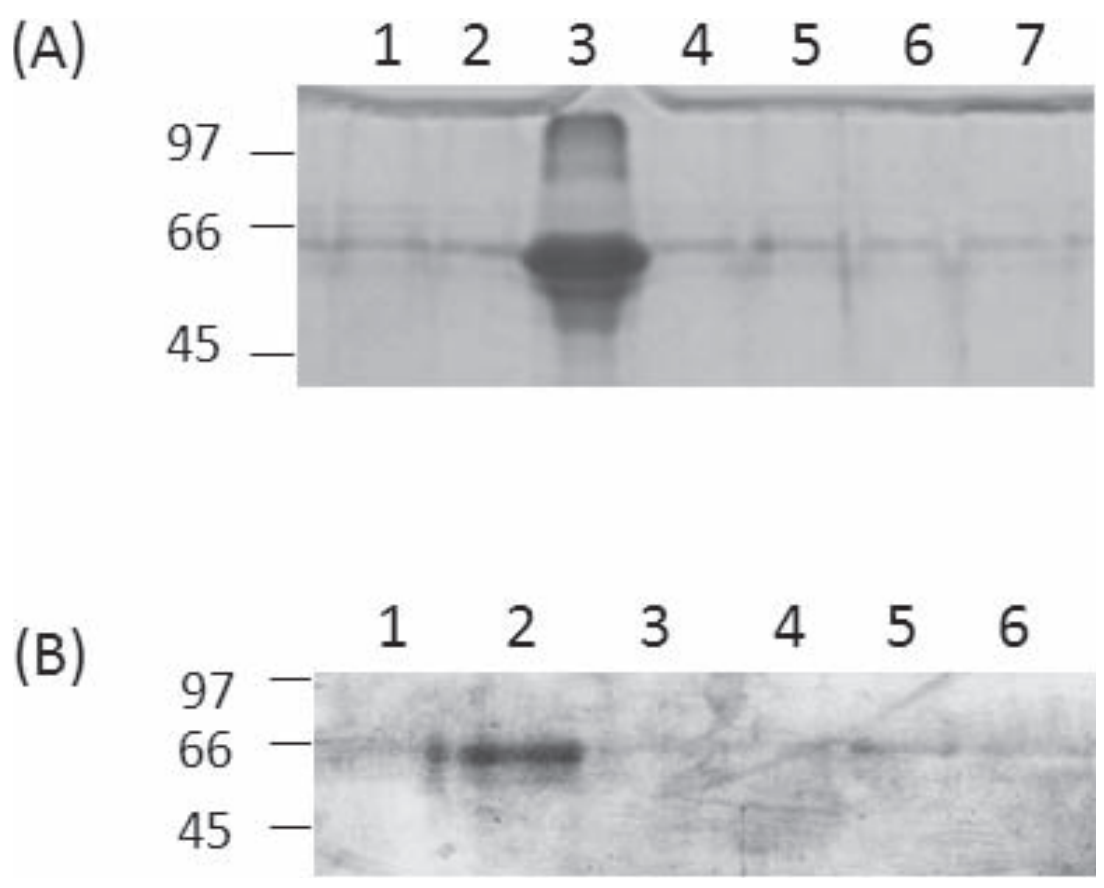

Fig. 3. Phospholipid Specificity of p62 Binding to Lipid Vesicles

(A) Purified p62 was mixed with lipid vesicles composed of phospholipid/PC/cholesterol $(5: 45: 50)$, and the mixture was incubated at $37^{\circ} \mathrm{C}$ for $30 \mathrm{~min}$. After centrifugation, the precipitates were analyzed by SDS-PAGE, followed by Coomassie brilliant blue staining. Lane 1, PC; lane 2, phosphatidic acid; lane 3, PG; lane 4, PS; lane 5, lysoPS; lane 6, phosphatidylinositol; lane 7, lysophosphatidylinositol. (B) Purified p62 was incubated at $37^{\circ} \mathrm{C}$ for $30 \mathrm{~min}$ with vesicles composed of various polyglycerophospholipids to be tested, PC, and cholesterol $(5: 45: 50)$. Lane 1 , PC as control; lane 2, PG; lane 3, cardiolipin; lane 4, bis(monoacylglycero)phosphate; lane 5, monolysocardiolipin; lane 6, dilysocardiolipin.

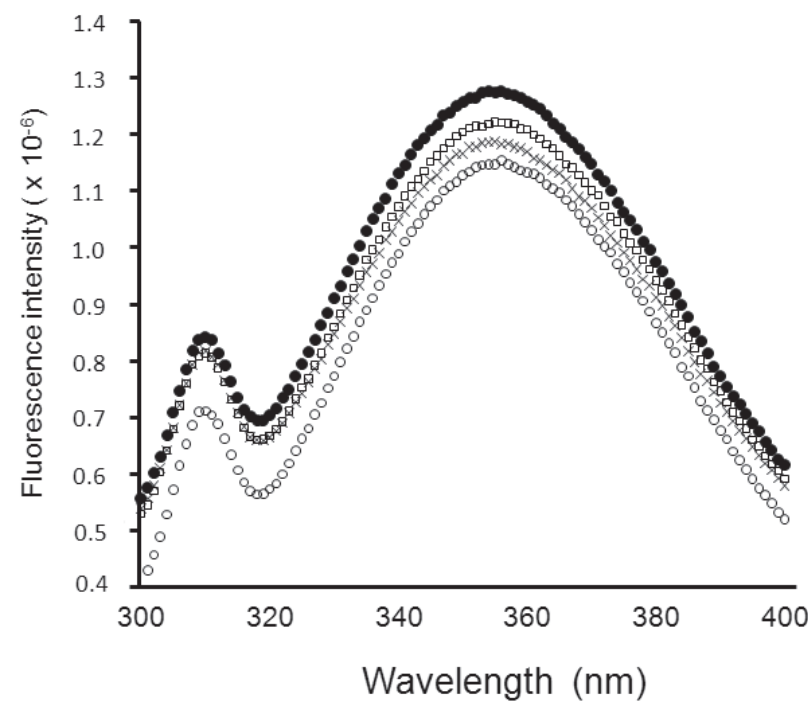

Fig. 4. Changes in Intrinsic Fluorescence Emission Spectra of p62 after Interaction with PG-containing Vesicles

p62 was incubated with PG/PC/cholesterol $(10: 40: 50, \bullet), \mathrm{PS} /$ $\mathrm{PC} /$ cholesterol (10:40:50, ), cardiolipin/PC/cholesterol (10:40: 50, $\times), \mathrm{PC} /$ cholesterol $(50: 50, \bigcirc)$ for $30 \mathrm{~min}$ at $37^{\circ} \mathrm{C}$. Fluorescence spectra were obtained at the excitation wavelength of $280 \mathrm{~nm}$.

cles. By exploiting the binding specificity of $\mathrm{p} 62$ to PS/cholesterol, p62 was purified to near homogeneity. The purified p62 specifically bound to
PG/PC/cholesterol $(5: 45: 50)$ vesicles, but not to $\mathrm{PS} / \mathrm{PC} /$ cholesterol $(5: 45: 50)$ vesicles. Since the purified p62 could bind to PS/cholesterol vesicles (50:50, data not shown), the possibility of alterations in binding specificity of p62 due to the purification procedures is excluded. It was suggested that a high density of PS in liposomal membranes was required for binding with $\mathrm{p} 62$.

Although p62 specifically bound to PG/PC/cholesterol $(5: 45: 50)$ vesicles, lipid vesicles composed of other acidic phospholipids, such as PS, phosphatidylinositol, or phosphatidic acid/PC/cholesterol $(5: 45: 50)$, did not interact with p62. Among the polyglycerophospholipid analogs examined, only PG-containing vesicles were able to bind to p62. These data suggest that p62 may recognize a molecular structure on the unesterified glycerol moiety of PG.

PG is found in almost all bacterial membranes as well as in the mitochondrial inner membranes of eukaryotic cells, ${ }^{23)}$ and is the second most abundant of the surfactant lipids. ${ }^{24,25)}$ It is also known that PG is required for the formation and function of thylakoid membranes in cyanobacteria and plants. ${ }^{26-28)}$ Although appreciable evidence for the essential role of PG in these cells is now accumu- 


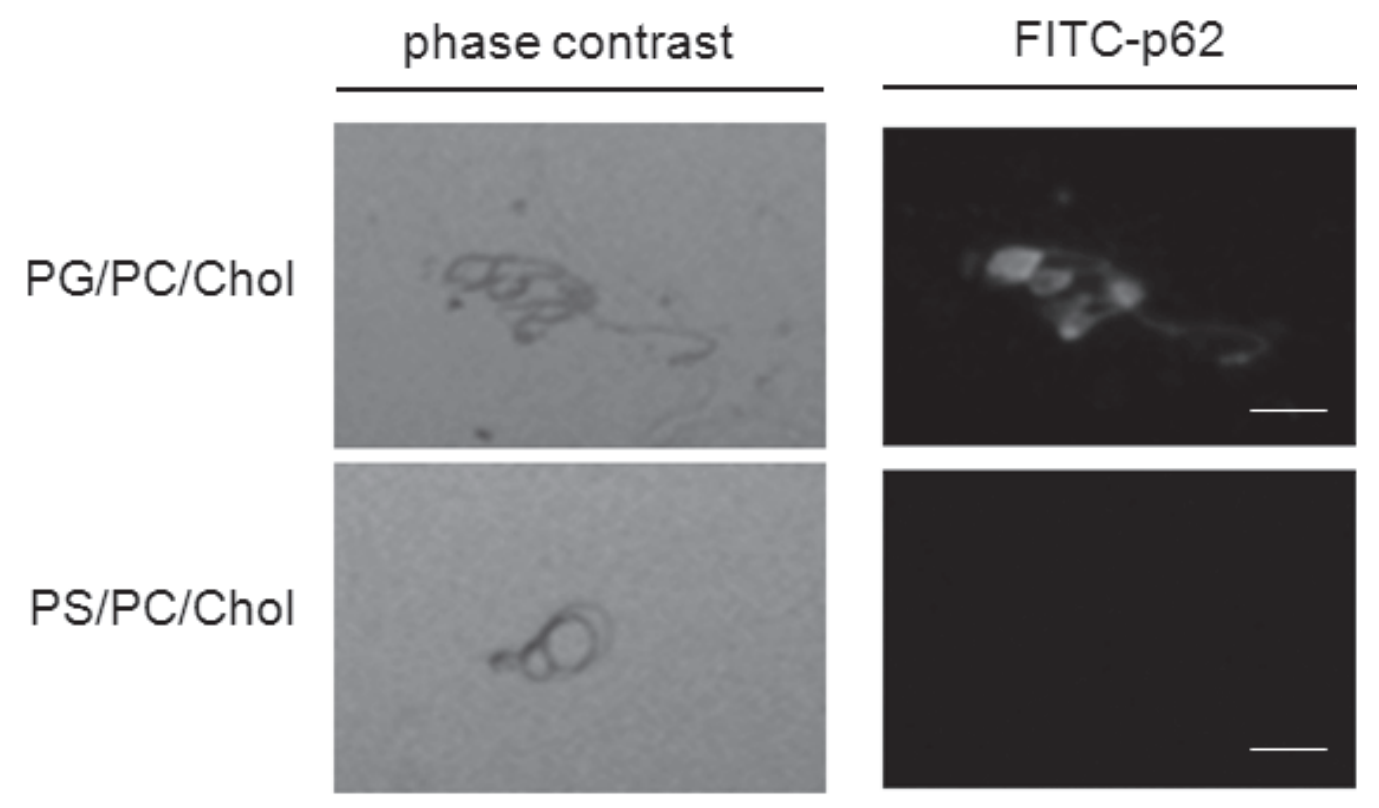

Fig. 5. Fluorescence Microscope Images of the Binding of FITC-labeled p62 to Giant Unilamellar Vesicles Containing PG

After incubation of giant unilamellar vesicles consisting of PG/POPC/cholesterol (10:40:50) or PS/POPC/cholesterol (10:40:50) with the FITClabeled p62 at room temperature, p62-bound vesicles were observed under a fluorescence microscope. The scale bar represents $10 \mu \mathrm{m}$.

lating, it is still insufficient to determine the exact role of PG in the membranes. We believe that p62 may be useful to define the precise roles of PG in biological membranes.

It is reported that tryptophan residues are involved in the interaction between several lipidbinding toxins and membranes. The tryptophan fluorescence of lysenin increases, and the maximum emission wavelength undergoes a blue shift after incubation with SM-containing vesicles. ${ }^{2,3)}$ In the present study, we observed similar changes in tryptophan fluorescence of $\mathrm{p} 62$ in the presence of PGcontaining membranes. This result suggests that tryptophan residues of p62 migrate to a less polar environment by interacting with PG molecules in membranes.

Giant unilamellar vesicles, which are readily observed under a microscope, serve as useful cell models. In this study, we observed that the interaction of FITC-labeled p62 with PG-containing giant liposomes but not with PS-containing giant liposomes. Membrane disruption activity of p62 was not observed under the conditions used. Although further analyses of the binding properties between p62 and PG are required, our findings suggest that p62 is a useful tool to probe the motion and function of PG in biological membranes.

\section{REFERENCES}

1) Yamaji, A., Sekizawa, Y., Emoto, K., Sakurabai, H., Inoue, K., Kobayashi, H. and Umeda, M. (1998) Lysenin, a novel sphingomyelin-specific binding protein. J. Biol. Chem., 273, 5300-5306.

2) Yamaji-Hasegawa, A., Makino, A., Baba, T., Senoh, Y., Kimura-Suda, H., Sato, S. B., Terada, N., Ohno, S., Kiyokawa, E., Umeda, M. and Kobayashi, T. (2003) Oligomerization and pore formation of a sphingomyelin-specific toxin, lysenin. J. Biol. Chem., 278, 22762-22770.

3) Kiyokawa, E., Makino, A., Ishii, K., Otsuka, N., Yamaji-Hasegawa, A. and Kobayashi, T. (2004) Recognition of sphingomyelin by lysenin and lysenin-related proteins. Biochemistry, 43, 97669773.

4) Shogomori, H. and Kobayashi, T. (2008) Lysenin: A sphingomyelin specific pore-forming toxin. Biochim. Biophys. Acta, 1780, 612-618.

5) Emoto, K., Kobayashi, T., Yamaji, A., Aizawa, H., Yahara, I., Inoue, K. and Umeda, M. (1996) Redistribution of phosphatidylethanolamine at the cleavage furrow of dividing cells during cytokinesis. Proc. Natl. Acad. Sci. U.S.A., 93, 12867-12872.

6) Emoto, K., Toyama-Sorimachi, N., Karasuyama, H., Inoue, K. and Umeda, M. (1997) Exposure of phosphatidylethanolamine on the surface of apoptotic cells. Exp. Cell Res., 232, 430-434.

7) Fujimoto, K., Umeda, M. and Fujimoto, T. (1996) 
Transmembrane phospholipid distribution revealed by freeze-fracture replica labeling. J. Cell Sci., 109, 2453-246018.

8) Umeda, M., Igarashi, K., Nam, K. S. and Inoue, K. (1989) Effective production of monoclonal antibodies against phosphatidylserine: stereo-specific recognition of phosphatidylserine by monoclonal antibody. J. Immunol., 143, 2273-2279.

9) Igarashi, K., Kaneda, M., Yamaji, A., Saido, T. C., Kikkawa, U., Ono, Y., Inoue, K. and Umeda, M. (1995) A novel phosphatidylserine-binding peptide motif defined by an anti-idiotypic monoclonal antibody. J. Biol. Chem., 270, 29075-29078.

10) Miyazawa, A., Umeda, M., Horikoshi, T., Yanagisawa, K., Yoshioka, T. and Inoue, K. (1988) Production and characterization of monoclonal antibodies that bind to phosphatidylinositol 4,5-bisphosphate. Mol. Immunol., 25, 1025-1031.

11) Ito, E., Miyazawa, A., Takagi, H., Yoshioka, T., Horikoshi, T., Yanagisawa, K., Nakamura, T., Kudo, Y., Umeda, M., Inoue, K. and Mikoshiba, K. (1991) Developmental assembly of calciummobilizing systems for excitatory amino acids in rat cerebellum. Neurosci. Res., 11, 179-188.

12) Bernheimer, A. W. and Avigad, L. S. (1979) A cytolytic protein from the edible mushroom, Pleurotus ostreatus. Biochim. Biophys. Acta, 585, 451-461.

13) Tomita, T., Noguchi, K., Mimuro, H., Ukaji, F., Ito, K., Sugawara-Tomita, N. and Hashimoto, Y. (2004) Pleurotolysin, a novel sphingomyelin-specific twocomponent cytolysin from the edible mushroom Pleurotus ostreatus, assembles into a transmembrane pore complex. J. Biol. Chem., 279, 2697526982.

14) Berne, S., Križaj, I., Pohleven, F., Turk, T., Maček, P. and Sepčić, K. (2002) Pleurotus and Agrocybe hemolysins, new proteins hypothetically involved in fungal fruiting. Biochim. Biophys. Acta, 1570, 153159.

15) Sepčić, K., Berne, S., Potrich, C., Turk, T., Maček, P. and Menestrina, G. (2003) Interaction of ostreolysin, a cytolytic protein from the edible mushroom Pleurotus ostreatus, with lipid membranes and modulation by lysophospholipids. Eur. J. Biochem., 270, 1199-1210.

16) Rebolj, K., Batistab, U., Sepčić, K., Cestnikc, V., Maček, P. and Frangež, R. (2007) Ostreolysin affects rat aorta ring tension and endothelial cell viability in vitro. Toxicon, 49, 1211-1213.

17) Sakurai, N., Kaneko, J., Kamio, Y. and Tomita, T. (2004) Cloning, expression, and pore-forming properties of mature and precursor forms of pleurotolysin, a sphingomyelin-specific two-component cytolysin from the edible mushroom Pleurotus ostreatus. Biochim. Biophys. Acta, 1679, 65-73.

18) Sepčić, K., Berne, S., Rebolja, K., Batistab, U., Plemenitasc, A., Sentjurcd, M. and Maček, P. (2004) Ostreolysin, a pore-forming protein from the oyster mushroom, interacts specifically with membrane cholesterol-rich lipid domains. FEBS Lett., 575, 8185.

19) Berne, S., Sepčić, K., Anderluh, G., Turk, T., Maček, P. and Ulrih, N. P. (2005) Effect of pH on the pore forming activity and conformational stability of ostreolysin, a lipid raft-binding protein from the edible mushroom Pleurotus ostreatus. Biochem. J., 44, 11137-11147.

20) Rebolj, K., Ulrih, N. P., Maček, P. and Sepčić, K. (2006) Steroid structural requirements for interaction of ostreolysin, a lipid-raft binding cytolysin, with lipid monolayers and bilayers. Biochim. Biophys. Acta, 1758, 1662-1670.

21) Akashi, K., Miyata, H., Itoh, H. and Kinosita, K., Jr. (1996) Preparation of giant liposomes in physiological conditions and their characterization under an optical microscope. Biophys. J., 71, 3242-3250.

22) Vera, J. C. and Rivas, C. (1988) Fluorescent labeling of nitrocellulose-bound protein at the nanogram level without changes in immunoreactivity. Anal. Biochem., 173, 399-404.

23) Matsumoto, K. (2001) Dispensable nature of phosphatidylglycerol in Escherichia coli: dual roles of anionic phospholipids. Mol. Microbiol., 39, 14271433.

24) Wu, Y., Medjane, S., Chabot, S., Kubrusly, F. S., Raw, I., Chignard, M. and Touqui, L. (2003) Surfactant protein-A and phosphatidylglycerol suppress type IIA phospholipase $\mathrm{A}_{2}$ synthesis via nuclear factor- $\kappa$ B. Am. J. Respir. Crit. Care Med., 168, 692699.

25) Numata, M., Chu, H. W., Dakhama, A. and Voelker, D. R. (2010) Pulmonary surfactant phosphatidylglycerol inhibits respiratory syncytial virus-induced inflammation and infection. Proc. Natl. Acad. Sci. U.S.A., 107, 320-325.

26) Hagio, M., Sakurai, I., Sato, S., Kato, T., Tabata, S. and Wada, H. (2002) Phosphatidylglycerol is essential for the development of thylakoid membranes in Arabidopsis thaliana. Plant Cell Physiol., 43, 14561464.

27) Frentzen, M. (2004) Phosphatidylglycerol and sulfoquinovosyldiacylglycerol: anionic membrane lipids and phosphate regulation. Curr. Opin. Plant Biol., 7, 270-276.

28) Laczkó-Dobos, H., Ughy, B., Tóth, S. Z., Komenda, J., Zsiros, O., Domonkos, I., Párducz, Á., Bogos, B., 
Komura, M., Itoh, S. and Gombos, Z. (2008) Role of phosphatidylglycerol in the function and assembly of photosystem II reaction center, studied in a $c d s A$ - inactivated PAL mutant strain of Synechocystis sp. PCC6803 that lacks phycobilisomes. Biochim. Biophys. Acta, 1777, 1184-1194. 